\title{
Nitric oxide production is associated to increased lipoperoxidation and active caspase- 3 in demyelinated brain regions of the taiep rat
}

\author{
Guadalupe Soto-Rodríguez ${ }^{1}$, Daniel Martínez-Fong ${ }^{2}$, Rosa Arroyo ${ }^{3}$, Patricia Aguilar-Alonso ${ }^{1}$, \\ Hector Rubio ${ }^{4}$, José Ramón Eguibar ${ }^{3}$, Araceli Ugarte ${ }^{3}$, Maricela Torres-Soto ${ }^{1}$, \\ Juan Antonio González-Barrios ${ }^{5}$, Jorge Cebada ${ }^{6 *}$, Eduardo Brambila ${ }^{1}$, Bertha Alicia Leon-Chavez ${ }^{1}$ \\ ${ }^{1}$ Facultad de Ciencias Químicas, Benemérita Universidad Autónoma de Puebla, San Claudio, México \\ ${ }^{2}$ Departamento de Fisiología, Biofísica y Neurociencias, Cinvestav, México D.F., México \\ ${ }^{3}$ Instituto de Fisiología, Benemérita Universidad Autónoma de Puebla, San Claudio, México \\ ${ }^{4}$ Facultad de Medicina, Universidad Autónoma de Yucatán, Mérida, Mexico \\ ${ }^{5}$ División de Medicina Genómica, Hospital Regional $1^{\circ}$ de Octubre, ISSSTE, Avenida Instituto Politécnico Nacional, México D. F., \\ México \\ ${ }^{6}$ Escuela de Biología, Benemérita Universidad Autónoma de Puebla, San Claudio, Mexico. \\ Email: ${ }^{*}$ alileon04@yahoo.com.mx
}

Received 15 August 2012; revised 20 September 2012; accepted 30 September 2012

\begin{abstract}
We previously showed that the increase in nitric oxide (NO) levels and NO synthase (NOS) expression correlate with the progression of reactive astrocytosis and demyelination in the brains of 6-month-old taiep rats. Increased levels of NO can result in high concentration of peroxynitrite and thus cause tissue damage, which consists of lipoperoxidation of the cytoplasmic membrane, such as the myelin, and of apoptotic and necrotic cell-death. On this basis, we studied whether the increased NO production is associated with lipoperoxidation and cell death in the cerebellum and brainstem over the age $(1,3,6$, and 8 months) of taiep rats. The results were compared with those obtained in matched Sprague-Dawley (SD) rats. We measured the levels of nitrites (NO production), malonyldialdehyde, and 4-hydroxyalkenal (lipoperoxidation) in brain tissue homogenates. The three NOS isoforms and cleaved caspase-3 were evaluated by using ELISA and immunostaining techniques. Our results showed that NO production and lipoperoxidation increased in the cerebellum and brainstem as the age of the taiep rats increased compared to SD rats. The overexpression of nNOS and iNOS were in the Purkinje cells, magnocellular neurons, and in oligodendrocytes, whereas the glial cells showed strong cleaved-caspase-3 immunoreactivity. In summary our results suggest that NO plays a role in the demyelination and cell death in the taiep rat.
\end{abstract}

"Corresponding author.
Keywords: Hypomyelination; Nitrite; Malonyldialdehyde; Apoptosis, Necrosis

\section{INTRODUCTION}

The taiep rat is a myelin mutant with a long survival time. The taiep rat develops a progressive neurological syndrome characterized by tremor, ataxia, immobility episodes, audiogenic seizures, and hindlimb paralysis [1]. In addition, taiep rats show hypomyelination at birth and progressive demyelination that leads to a highly hypomyelinated central nervous system (CNS) in adult-hood $[2,3]$. The deficit in myelin has been directly associated with a cytoskeleton alteration of oligodendrocytes, which show an accumulation of microtubules in their soma $[4,5]$, and with changes in expression and intracellular location of myelin-gene products [6,7]. To date, the genetic mutation causing the myelin defect in the taiep rat remains unknown. It is believed to be of a multifactorial type. In the taiep rat, contrary to the expected oligodendrocyte death, it has been found an apparent increase in the number of oligodendrocytes at least in some regions of the central nervous system such as funiculi of the spinal cord, the optic nerve and the cerebellum [2]. To date, there is no evidence of oligodendrocyte death in the taiep rat.

Previous studies in the taiep rat have shown an increase of nitric oxide (NO) levels and upregulation of the mRNA of the nitric oxide synthases (NOS) in cultured glial cells when stimulated by lipopolysaccharides or the tumor growth factor (TNF) [8], and in homogenates from 
the cerebellum of 6-month-old rats [9]. The increased levels of NO have been related to apoptosis [10] and necrosis of neurons $[11,12]$, caused by the production of peroxynitrite [10,12-14]. Accordingly, the inhibition of the production of NO by administration of N-nitro-Larginine methyl ester (L-NAME) and of superoxide by the addition of superoxide dismutase decrease lipoperoxidation, apoptosis, and the infarct zone size in several models of disease [11,14,15]. In contrast, the increase of nitrate/nitrite levels in serum, lipoperoxidation products (malonyldialdehyde plus 4-hydroxyalkenals), and glutathione peroxidase activity have been associated with remitting multiple sclerosis as shown in patients of a Mexican population [16]. Both the neuronal (n)NOS and the inducible (i)NOS have also been associated with the loss of oligodendrocytes and demyelination [17-19]. It is known that the iNOS is not significantly expressed by resident cells unless cellular activation occurs. In this case, the iNOS is produced by several types of cells, such as macrophages, microglia, astrocytes [20,21], oligodendrocytes [22], and even cerebral endothelial cells [23].

The oligodendrocyte cell death is mediated by caspases in the pathogenesis of autoimmune demyelination diseases such as multiple sclerosis (MS). The inhibition of caspase-3 activation in oligodendrocytes decreased apoptosis [24]. In addition, other caspases are involved in the inflammatory and apoptotic processes in oligodendrocytes. Accordingly, the caspase-11 and caspase1 mRNA and protein levels are significantly elevated in oligodendrocytes in both acute and chronic MS [24-26]. In support of this, the number of apoptotic oligodendrocytes and severity of experimental allergic encephalomyelitis (EAE) are reduced in caspase-1- or caspase11-deficient animals [25].

These antecedents clearly demonstrate the participation of NO through peroxynitrite in lipoperoxidation and oligodendrocyte death by necrosis or apoptosis mechanisms. On this basis, our work aims to demonstrate that the increased NO production is associated with lipoperoxidation and cell death in the cerebellum and brainstem of taiep rats. We analyzed the production of $\mathrm{NO}$ as assessed by nitrite accumulation, the expression of the three NOS isoforms by using an enzyme-linked immunosorbent assay (ELISA), and the indirect immunofluorescence in the brainstem and cerebellum at ages of 1, 3, 6 , and 8 months. In addition, the levels of malondialdehyde-4-hydroxyalkenals and caspase- 3 immunoreactivity (IR) were determined and used as the markers of cell death. Our results provided evidence of the possible role of $\mathrm{NO}$ in the neuron death of necrotic type and demyelination in the taiep rat, a model of the hypomyelination-demyelination process.

\section{MATERIALS AND METHODS}

\subsection{Experimental Animals}

Taiep rats, ages from 1 to 8 months, were obtained from the vivarium of the Institute of Physiology, BUAP and aged-matched Sprague-Dawley rats were from the CINVESTAV. Animals were maintained in adequate animal rooms with controlled conditions of temperature $\left(22^{\circ} \mathrm{C} \pm 1^{\circ} \mathrm{C}\right)$ and light-dark cycle $(12 \mathrm{~h}: 12 \mathrm{~h}$ light-dark; light onset at 0700). Food and water were provided ad libitum. All procedures were in accordance with the Mexican current legislation, the NOM-062-ZOO-1999 (SAGARPA), based on the Guide for the Care and Use of Laboratory Animals, NRC. The Institutional Animal Care and Use Committee (IACUC) approved our animal-use procedures with the protocol number 410. All efforts were made to minimize animal suffering.

\subsection{Nitric Oxide Determination}

The cerebellum and brainstem of control or taiep rats ( $n$ $=5$ in each group) were mechanically homogenized in phosphate-buffered saline solution, $\mathrm{pH} 7.4$ (PBS) and centrifuged at $12,500 \mathrm{rpm}$ for $30 \mathrm{~min}$ at $4^{\circ} \mathrm{C}$ by using a 17TR microcentrifuge (Hanil Science Industrial Co, Ltd; Inchun, Korea). The NO production was assessed by the accumulation of nitrites $\left(\mathrm{NO}_{2}^{-}\right)$in supernatants of homogenates as described elsewhere [8,27]. Briefly, the nitrite concentration in $100 \mu \mathrm{L}$ of supernatant was measured by using a colorimetric reaction generated by the addition of $100 \mu \mathrm{L}$ of Griess reagent, which was composed of equal volumes of $0.1 \% \mathrm{~N}$-(1-naphthyl) ethylenediamine dihydrochloride and $1.32 \%$ sulfanilamide in $60 \%$ acetic acid. The absorbance of the samples was determined at $540 \mathrm{~nm}$ with a SmartSpec 3000 spectrophotometer (Bio-Rad, Hercules, CA, USA) and interpolated by using a standard curve of $\mathrm{NaNO}_{2}(1$ to $10 \mu \mathrm{M})$ to calculate the nitrite content.

\subsection{Enzyme-Linked Immunosorbent Assay}

ELISA was used to determine the three isoforms of NOS in homogenates of the cerebellum and brainstem of control or taiep rats ( $n=5$ in each group) as we described previously $[8,28]$. The protein content was determined using the method described elsewhere [29]. Aliquots containing $5 \mu \mathrm{g}$ of total protein were placed into wells of the ELISA plates for the separate determination of nNOS, iNOS, and eNOS. Volumes of $100 \mu \mathrm{L}$ of $0.1 \mathrm{M}$ carbonate buffer were added into each well and the plate was incubated for $18 \mathrm{~h}$ at $4^{\circ} \mathrm{C}$. To block nonspecific binding sites, $200 \mu \mathrm{L}$ of $0.5 \%$ bovine serum albumin, IgG free, was added to each well at room temperature (RT). After a 30-min incubation, the wells were washed three times with PBS-Tween $20(0.1 \%)$. Mouse monoclonal anti- 
bodies to nNOS, iNOS, or eNOS (1:200 dilution; SigmaAldrich; St. Louis, MO, USA) were added into each well and incubated for $2 \mathrm{~h}$ at RT. After three washings with PBS, a horseradish-peroxidase-conjugated goat antimouse IgG (1:1000 dilution; Dako A/S Denmark Dako North America, Inc. Carpinteria, CA) was added and incubated for $2 \mathrm{~h}$ at RT. The antibody-antigen complex was revealed by adding $100 \mu \mathrm{L}$ of ABTS containing $0.3 \% \mathrm{H}_{2} \mathrm{O}_{2}$ into each well. After $15 \mathrm{~min}$, the optical density (OD) was determined at $415 \mathrm{~nm}$ using a Benchmark multiplate reader (Bio-Rad, Hercules, CA, USA) as described previously [8].

\subsection{Immunolabeling of NOS and Cleaved Caspase-3}

The cell location of NOS isoforms was analyzed by double immunofluorescence to specific NOS and galactocerebroside (oligodendrocyte) or glial fibrillary acidic protein (GFAP; astroglial cells) in sagital brain slices of control and taiep rats ( $n=3$ in each group). Rats were deeply anesthetized with chloral hydrate and perfused through the ascending aorta with $100 \mathrm{~mL}$ of PBS, followed by $150 \mathrm{~mL}$ of $4 \%$ paraformaldehyde in PBS. Their brains were removed and maintained in the fixative for $48 \mathrm{~h}$ at $4^{\circ} \mathrm{C}$. After overnight maintenance in PBS containing $10 \%$ sucrose at $4^{\circ} \mathrm{C}$, each brain was frozen and sectioned into $35-\mu \mathrm{m}$ slices on the sagittal plane using a Leitz cryostat (LEICA 2000R). Slices were individually collected in a 24-well plate containing PBS and used for fluorescent immunolabeling of oligodendrocyte (galactocerebroside), astroglial cells (GFAP), and NOS isoforms. Slices were incubated with $0.5 \% \mathrm{IgG}$-free bovine serum albumin in PBS-Tween-20 (0.1\%) for $20 \mathrm{~min}$ at room temperature. The primary antibodies were mouse monoclonal antibodies to nNOS, iNOS, or eNOS (1:200 dilution; Sigma-Aldrich; St. Louis, MO, USA), a mouse monoclonal antibody to galactocerebroside (Galc), and a rabbit polyclonal antibody to GFAP. The secondary antibodies were an IgG antimouse, made in goats, labeled with fluorescein and an $\operatorname{IgG}$ antirabbit, made in goats, labeled with rhodamine.

The slices were mounted on glass slides using Vectashield (Vector Laboratories; Burlington, Ontario, Canada) and analyzed on a confocal imaging system equipped with a krypton-argon laser beam (Bio-Rad MRC-600, Watford, UK) as we have described elsewhere [9]. The fluorescence was detected at Ex-Em wavelengths of 488 $522 \mathrm{~nm}$ (green channel) and 568 - $585 \mathrm{~nm}$ (red channel). Ten to twenty consecutive optical sections at $1 \mu \mathrm{m}$ intervals were obtained in the z-series. The resulting images were projected on a bidimensional plane and were overlapped on the screen monitor using green for FITC and red for Rho. The brain sections processed under similar conditions in the absence of the primary antibody were used as negative controls.

The cleaved caspase- 3 immunoreactivity was analyzed by an immunohistochemical method. The fixed brains were maintained overnight in PBS containing 30\% sucrose at $4^{\circ} \mathrm{C}$. Then, each brain was frozen and sectioned into $16 \mu \mathrm{m}$ slices on the coronal plane using a Leica SM100 cryostat (Leica Microsystems; Nussloch, Germany). Slices were individually collected in a 24 -well plate containing PBS and used for immunohistochemistry for cleaved caspase-3. The slices were incubated with PBS-Triton $(0.1 \%)$ and later with $10 \%$ horse serum in PBS-Triton $(0.1 \%)$ for $60 \mathrm{~min}$ at room temperature. The slices were incubated overnight with rabbit polyclonal antibody against cleaved caspasa-3 (1:300 dilution, Cell Signaling Technology, 3 Trask Lane, Danvers, MA 01923) and then with the secondary antibody biotinylated anti-rabbit $\operatorname{IgG}(\mathrm{H}+\mathrm{L})$, made in goats, $(1: 600$ dilution; Vector Laboratories. Burlingame, CA, USA) for 2 hours at room temperature. After rinsing, the slices were incubated with streptavadin-horseradish peroxidase conjugate (BRL Inc., Gaithersburg, MD), diluted 1:400, again for 30 minutes at room temperature. The peroxidase reaction was developed by immersion in a freshly prepared solution of $0.02 \% 3,3$ '-diaminobenzidine (DAB, Sigma). The slices were counterstained with cresyl violet. The caspase- 3 immunoreactivity was analyzed with magnification of $5 \times, 20 \times$, and $40 \times$ using a Leica DMIRE2 microscope (Leica Microsystems; Wetzlar, Germany). Images were digitalized with a Leica DC300F camera (Leica Microsystems; Nussloch, Germany) and analyzed with workstation Leica FW4000, version V1.2.1 (Leica Microsystems Vertrieb GmbH; Bensheim, Germany).

The histophathology study of the cerebellum and brainstem from the brains of each experimental group was analyzed by hematoxylin-eosin staining in coronary brain slices at 24-h postreperfusion ( $n=3$ in each group). Paraffin-embedded tissue sections of $5 \mu \mathrm{m}$ were stained with hematoxylin and eosin and examined at a magnifycation objective of 40× (Mod BM 1000, Jenopika Camera, Wetzlar; Leica, Germany). Digital photomicrographs were made from 5 randomly selected fields of each tissue section of each experimental group at 24-h postreperfusion (Progress capture pro 2.1, Leica).

\subsection{Lipoperoxidation}

Malonyldialdehyde (MDA) and 4-hydroxyalkenal (HA) were measured in supernatants of homogenates of the cerebellum and brainstem using the method described elsewhere [11]. The colorimetric reaction in $200 \mu \mathrm{L}$ of supernatant was caused by the subsequent addition of $0.650 \mathrm{~mL}$ of $10.3 \mathrm{mM} \mathrm{N}$-methyl-2phenyl-indole diluted in a mixture of acetonitrile:methanol (3:1). The reaction 
was started by the addition of $150 \mu \mathrm{L}$ of methanesulfonic acid. The reaction mixture was strongly vortexed and incubated at $45^{\circ} \mathrm{C}$ for $1 \mathrm{~h}$ and then centrifuged at 3000 rpm for $10 \mathrm{~min}$. The absorbance in the supernatant was read at $586 \mathrm{~nm}$ with a SmartSpec 3000 spectrophotometer (Bio-Rad; Hercules, CA, USA). The absorbance values were compared to a standard curve in the concentration range of 0.5 to $5 \mu \mathrm{M}$ of 1,1,3,3-tetramethoxypropane (10 $\mathrm{mM}$ stock) to calculate the malondialdehyde and 4-hydroxyalkenal contents in the samples.

\subsection{Statistical Analysis}

All values are the mean $\pm \mathrm{SE}$ obtained from at least 5 independent experiments. After testing for normality with the Snedecor F-analysis. The significance of differences were analyzed by an unpaired Student's $t$-test. The data were analyzed using the Graph pad prism software (version 5.00). The significance value was considered at $P<0.05$.

\section{RESULTS}

\subsection{NO Levels}

The production of NO was estimated by determining the nitrite content in supernatants of cerebellum and brainstem homogenates from 1,3,6, and 8-month-old taiep and control rats. In the cerebellum of taiep rats, the NO level increased by $95 \% \pm 2 \%$ at age 1 month and reached the maximum increase $(155 \% \pm 46 \%)$ at 3 months. Thereafter, the levels decreased but remained significantly high as long as age 8 months when compared with SD rats (Figure 1). In the brainstem, the production of NO in taiep rats significantly increased $73 \% \pm 19 \%$ at age 3 months, $60 \% \pm 23 \%$ at 6 months old, and $317 \% \pm$ $80 \%$ at 8 months old when compared with control (SD) rats (Figure 1) $(P<0.05)$.

\subsection{NOS Protein Levels}

The enhanced production of NO was related to an increase in protein levels of the three NOS isoforms in the cerebellum and brainstem of the taiep rat as assessed by ELISA. The increases were statistically significant when compared with values of the control SD rats and they were, in the cerebellum (Figure 2), $70 \% \pm 4 \%$ for nNOS at age 6 months, and $19 \% \pm 2 \%$ at 3 months, and $107 \% \pm$ $17 \%$ at 6 months for the iNOS. The values of the eNOS protein in the cerebellum did not show statistical differences over age when compared with values of SD rats (Figure 2). The percentage increases in the NOS protein levels in the brainstem (Figure 2) were 264\% $\pm 3 \%$ for nNOS at age 6 months, $193 \% \pm 13 \%$ at age 6 months for iNOS, but there was a decrease to $55 \% \pm 5 \%$ at 8 months.
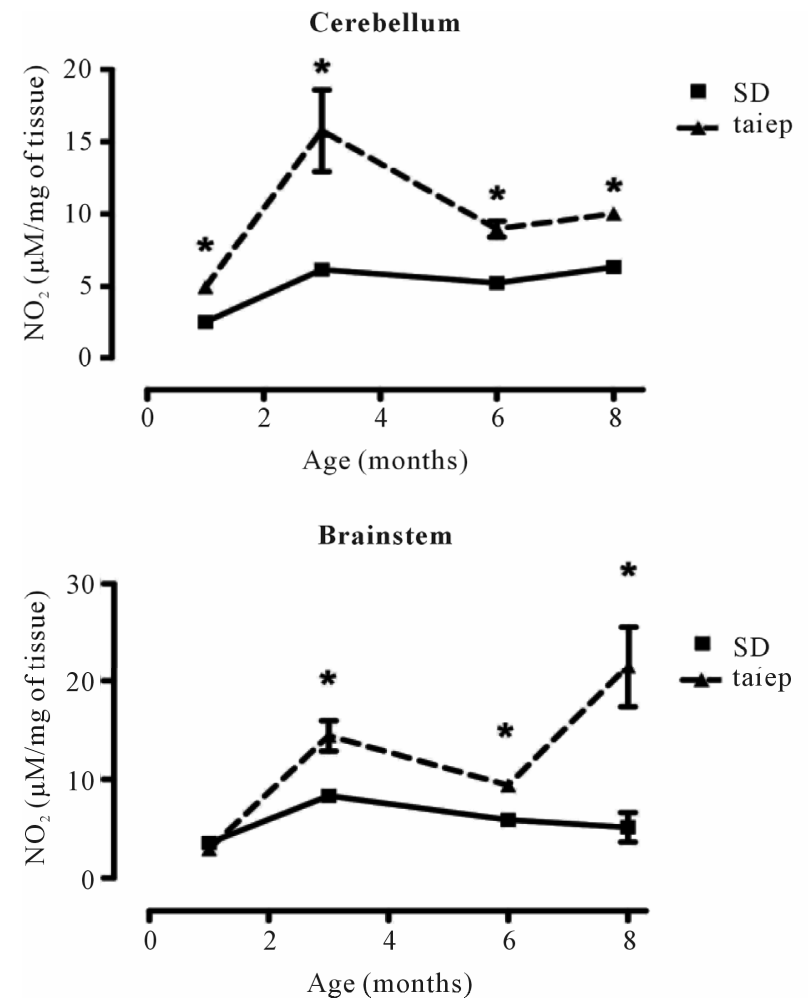

Figure 1. Increased nitric oxide production in the cerebellum and brainstem of the taiep rat. Nitrite levels were determined by using the Griess method. Each value is the mean $\pm \mathrm{SD}$ of 5 independent experiments made in triplicate. SD, SpragueDawley. ${ }^{*}$ Significantly different from the control group, $p<$ 0.05 , Student's $t$-test.

The increases in the eNOS levels in taiep rats were $32 \%$ $\pm 8 \%$ at age 3 months and $39 \% \pm 4 \%$ at 6 months when compared with SD rat values.

\subsection{Cell Location of NOS Isoforms}

Confocal microscopy and double immunofluorescence were used to determine the location of the NOS isoform immunoreactivity (IR) revealed by a secondary antibody fluorescein-labeled (green channel) in neuronal cell types and revealed by a secondary antibody rhodamine-labeled (red channel). The nNOS-IR was seen in magnocellular neurons and astrocytes in the brainstem (Figure 3) and in Purkinje cells in the cerebellum, as previously demonstrated by our group $[9,28]$. The iNOS-IR was located mainly in the magnocellular neurons, in oligodendrocytes (Figure 3), and in Purkinje cells, as previously demonstrated by our group [9], whereas the eNOS-IR was present in oligodendrocytes, astrocytes, and neurons (Figure 3). The IR intensity in the taiep rat was qualitatively higher than that in SD rats.

\subsection{Lipoperoxidation Levels}

The lipoperoxidation assayed by MDA + 4-HAD levels 

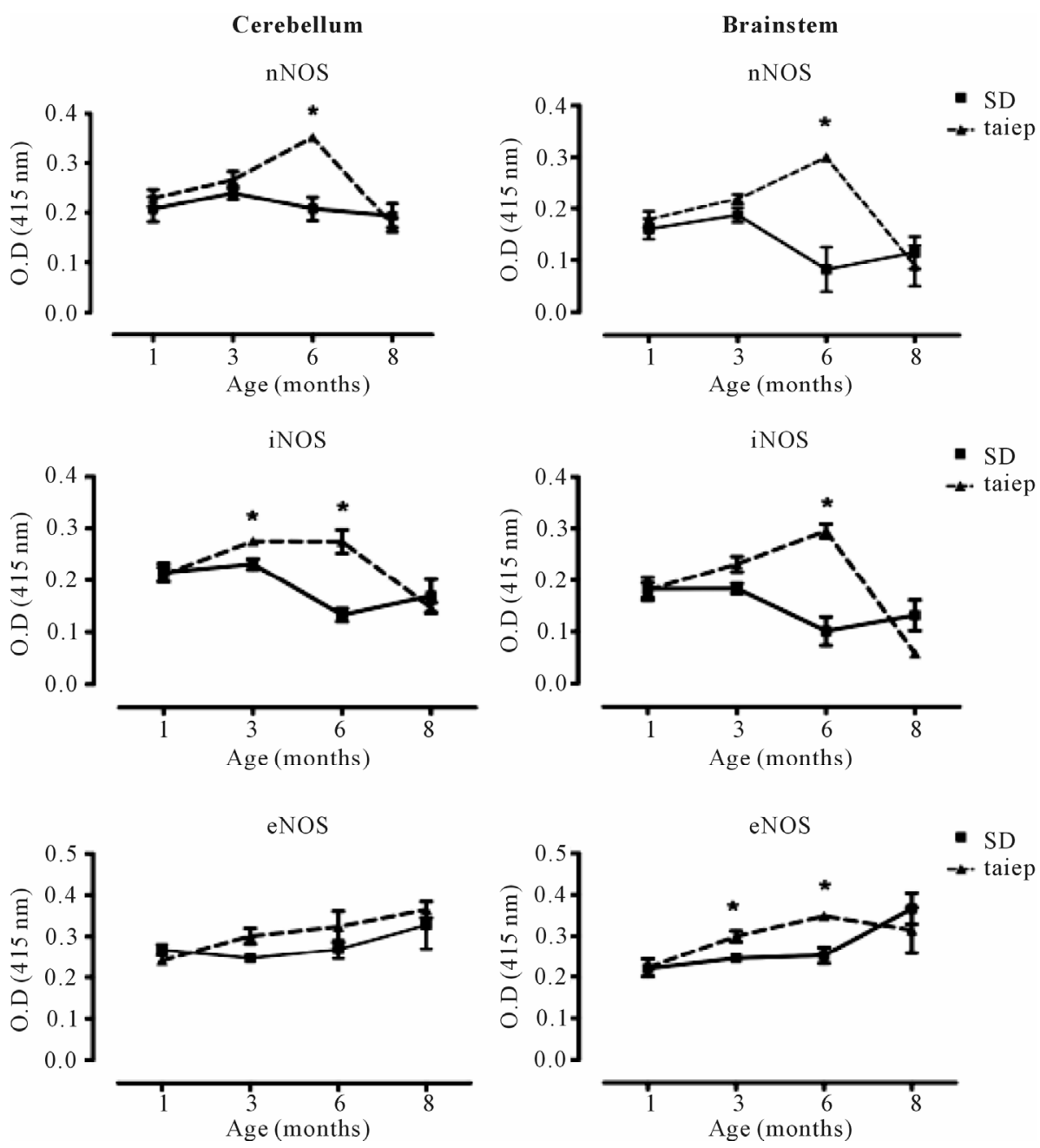

Figure 2. Levels of NOS isoform proteins in the cerebellum and brainstem of the taiep rat. ELISA was used to determine the protein levels of nNOS, iNOS, and eNOS in separate assays. Each value is the mean \pm SD of 5 independent experiments made in triplicate. SD, Sprague-Dawley. ${ }^{*}$ Significantly different from the control group, $P<0.05$, Student's $t$-test.

was significantly increased in the cerebellum and brainstem of the taiep rat as compared to control rats (Figure 4). In the cerebellum, the lipoperoxidation level increased from $142 \% \pm 20 \%$ at age 1 month to $325 \% \pm$ $41 \%$ at 3 months, and remained high up to age 8 months. In the brainstem of taiep rats, the increases of lipoperoxidation levels were $110 \% \pm 20 \%$ at 3 months, $33 \% \pm$ $5 \%$ at 6 months, and $196 \% \pm 42 \%$ at age 8 months.

\subsection{Cleaved Caspase-3 Immunoreactivity}

The cleaved caspase-3 IR in the cerebellum and brain stem significantly increased with age in the taiep rats as compared to that in matched control regions (Figure 5). The micrograph of the cerebellum from the one-monthold SD rat shows from top to bottom the molecular layer, the Purkinje layer (arrowhead), and the granular layer with the highest cell density. At the bottom right, a small area of white matter of the cerebellum appears. The cell morphology of the three layers of cerebellar cortex looks normal from ages 1 month to 8 months. The cleaved caspase-3 IR appears in some cells with morphology similar to glial cells around the Purkinje cells (arrows). It is interesting that the caspase-3 IR disappeared in the cerebellum of SD rats with ages from 3 months to 8 months.

In the taiep rat, the morphology of cerebellar cortex is diffusely defined. The Purkinje layer is not morphologically evident with Nissl staining at the age of one month. The immunoreactivity against cleaved caspase- 3 significantly increased with age in the granular layer and Purkinje layer. Some immunoreactive cells are also seen in the molecular layer, exhibiting morphological characteristics of glial cells at age 1 month. In older ages, some neurons were caspase-3 immunoreactive. The Purkinje cells (arrowheads) were positive to caspase-3 suggesting 

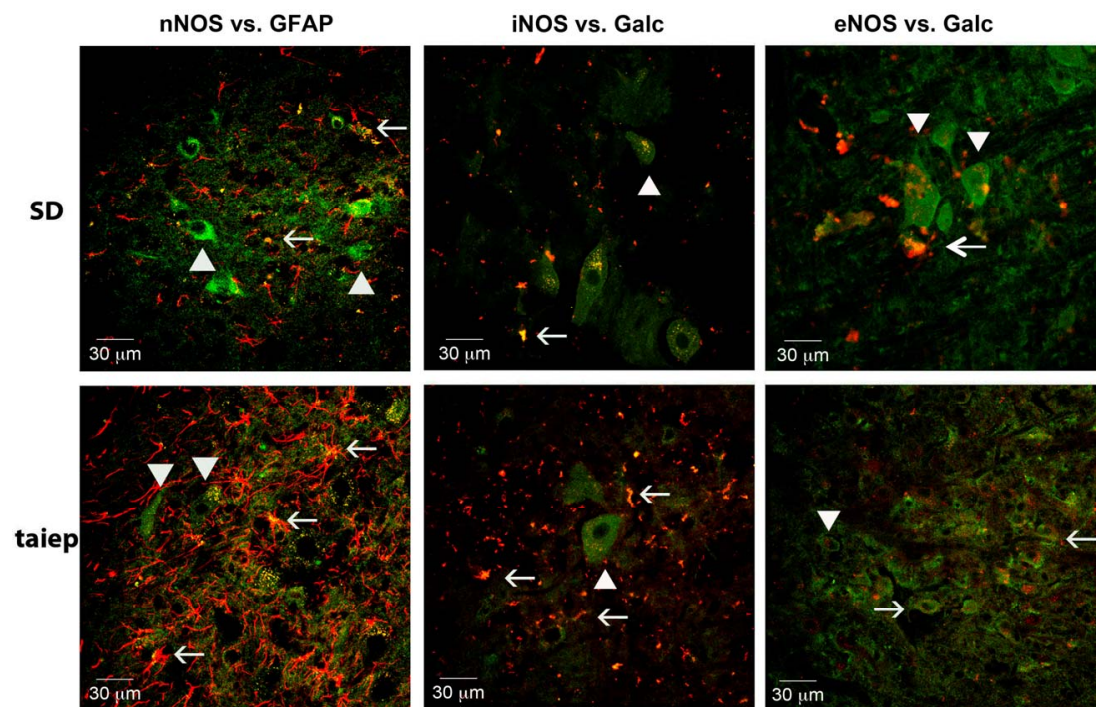

Figure 3. Merged confocal micrographs showing the cell location of the immunoreactivity of nNOS, iNOS, and eNOS in the brainstem of SD and taiep rats at age 3 months. The immunoreactivity (IR) for nNOS (green; arrowheads) was seen mainly on the magnocellular neurons and less in astrocytes (red; arrows) of control and taiep rats. The iNOS-IR (green; arrowheads) was in neurons and oligodendrocytes (red, arrows). The eNOS-IR (green; arrowheads) was in oligodendrocytes (red; arrows) and neurons (arrowheads). These micrographs are representative of three experiments. GFAP (glial fibrillary acidic protein); Galc (galactocerebroside); SD (Sprague-Dawley).

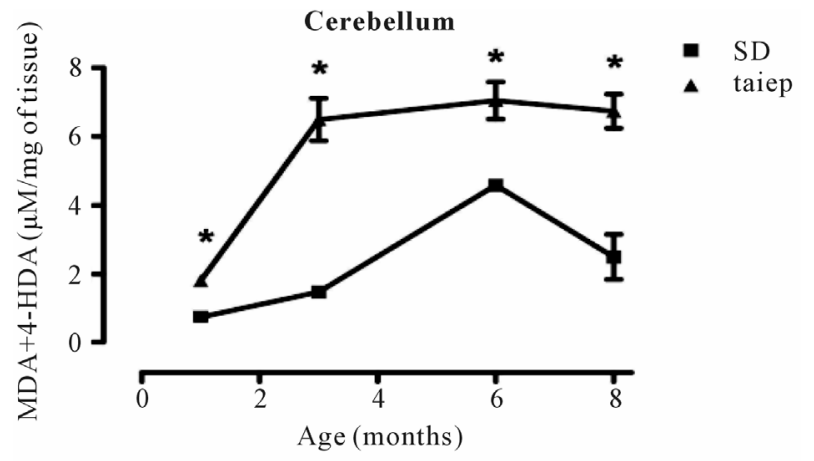

that the taiep damage has reached these cells at age 8 months. The density of neuropil in the molecular layer was also reduced in 6-month-old taiep rats when compared with that of a matched SD rat.

In the brainstem of SD rats, the magnocellular neurons (arrowhead) showed normal morphological characteristics from one month to eight months (Figure 5). Those neurons have well-defined neuritic projections, prominent nucleoli, and Nissl bodies. Around some glial cells, scarce and weak immunoreactivity against caspase- 3 is seen from age one to 8 months. In the brainstem of the taiep rat, the number of neurons (arrowhead) was less

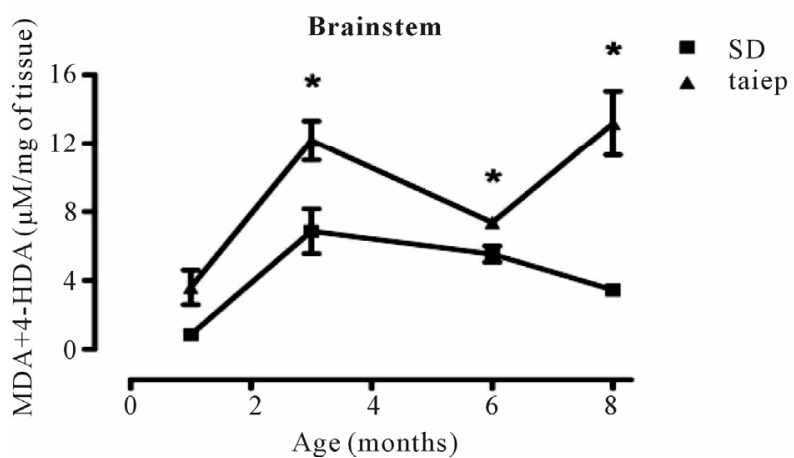

Figure 4. Levels of lipoperoxidation in the cerebellum and brainstem. The malonyldialdehyde (MDA) and 4-hydroxyalkenal (4-HAD) concentrations measured by using the GerardMonnier's method were used as biomarkers of lipoperoxidation. Each value is the mean \pm SD of 5 independent experiments made in triplicate. SD, Sprague-Dawley. "Significantly different from the control group, $P<0.05$, Student's $t$-test. than that of the matched control at one month, and their nuclei and nucleoli were well-defined. The number of neurons, their projections, and their intensity of the Nissl staining significantly decreased in older ages $(6$ and 8 months) of the taiep rats. The immunoreactivity against cleaved caspase-3 significantly increased over age in comparison with the control rats. There were abundant glial cells around neurons with high immunoreactivity to cleaved caspase-3 (arrows).

The slices of the cerebellum and brain stem of the SD and taiep rats age 8 months were stained with hematoxylin-eosin to gain further insight into the brain tissue damage caused by the taiep pathology. The hematoxylineosin staining revealed some abnormalities. First, there was a loss of normal morphology in the Purkinje neurons of cerebellum and magnocellular neurons of brainstem in 8-month-old taiep rats (Figure 6, arrowhead). Second, 

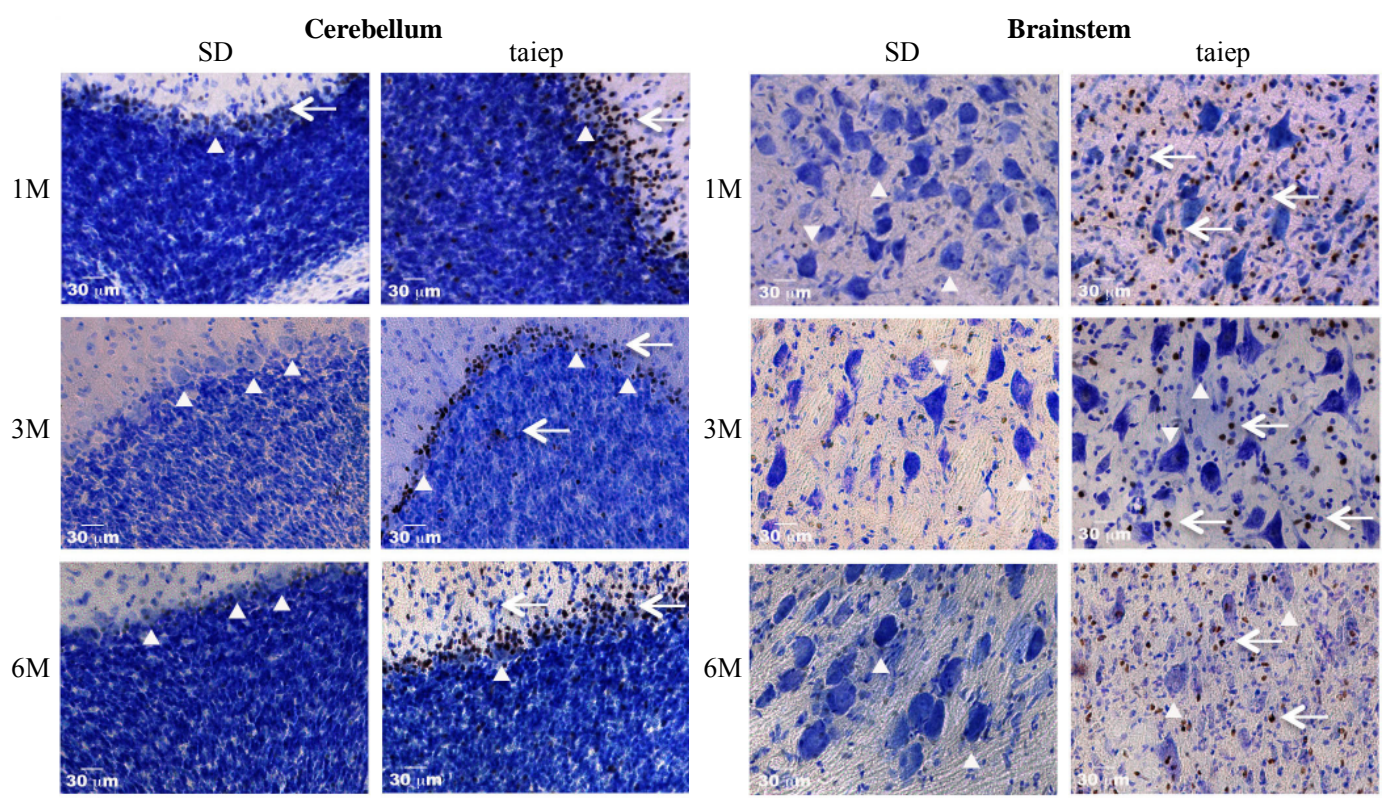

$8 \mathrm{M}$
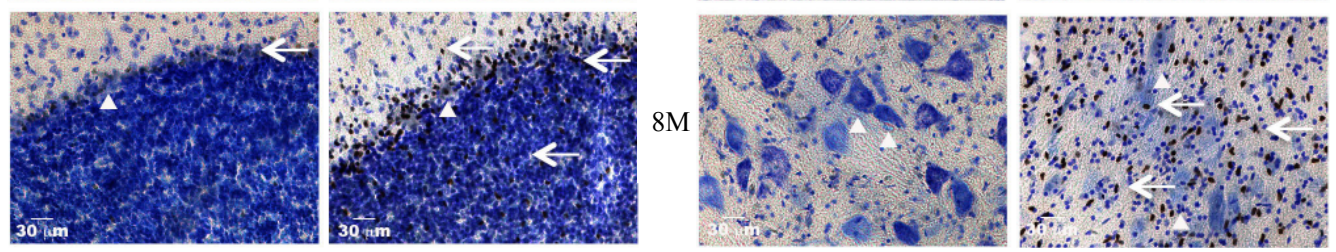

Figure 5. Immunohistochemistry against caspase-3 and Nissl counterstaining in slides of the cerebellum and brainstem at four different ages. The composite figures comparatively show micrographs of the cerebellum and brainstem from taiep and SD rats. The labels at the left side of the micrographs are ages of 1, 3, 6, and 8 months. The immunostaining against cleaved caspase- 3 is shown by the dark marks (arrows) and the Nissl stain appears in blue (arrowheads).
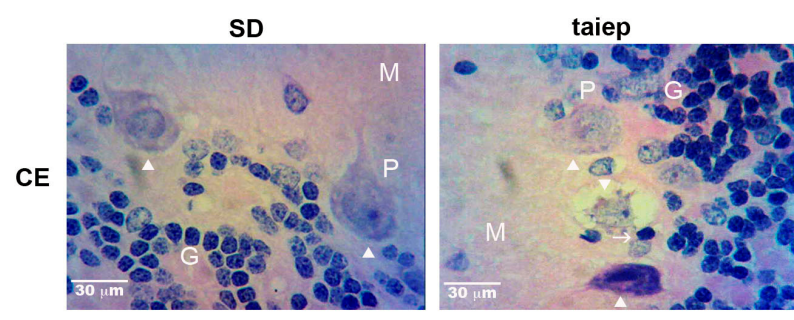

BS
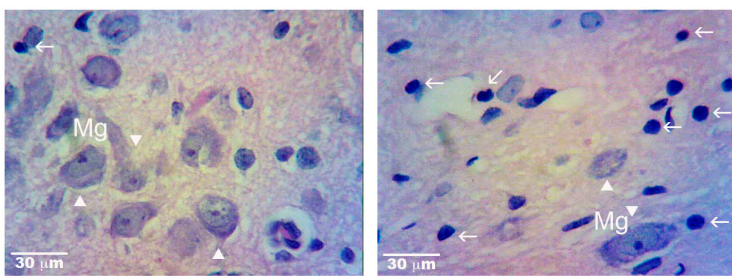

Figure 6. Hematoxylin-Eosin staining in the cerebellum and brainstem slides of taiep and control rats. The micrographs are representative of three experiments. Thin arrows are neurons and thick arrows are picnotic glial cells. CE, cerebellum; BS, brainstem; SD, Sprague-Dawley. P: Purkinje layer; G: granular layer and $\mathrm{M}$ : molecular layer and $\mathrm{Mg}$ : magnocellular neuron.

both Purkinje neurons and the magnocellular neurons of the taiep rat displayed a pale and diffuse staining, suggesting necrosis. Third, there are empty vacuoles or vacuoles with shrunken cells in the brain regions studied of the taiep rats. Finally, glial cells in the taiep rat are more abundant and with a picnotic nucleus, suggesting condensation of chromatin (Figure 6, arrows).

\section{DISCUSSION}

Our results strongly suggest that the increase in NO production is associated with lipoperoxidation and apoptosis in the cerebellum and brainstem, the most demyelinated brain regions [2,3] of the taiep rat from ages 3 to 8 months. The iNOS and nNOS isoforms, which also increased after 3 months, could be involved in the high production of NO. iNOS and nNOS expressed in the oligodendrocytes and neurons of old taiep rats might be participating in the death of those cells as shown by previous reports [9,25,30,31]. Accordingly, our immunohistochemistry against cleaved caspase- 3 and histopathology studies clearly showed the presence of apoptosis, necrosis, and reactive astrogliosis in the taiep rats.

The NO role is dual; first the NO exerts a protective function on neuronal [31-33] and glial cells and second it becomes cytotoxic at high and sustained concentrations [10-14]. Neurons in the Purkinje and granular layers of 
the cerebellum and of the magnocellular nuclei in the brainstem of the control and taiep rats expressed iNOS and nNOS. However, the large neurons, where iNOS and nNOS were overexpressed, died by a necrosis mechanism after age 6 months in the taiep rats, suggesting that the NO production by iNOS and nNOS might exert a protective effect on large neurons at ages younger than 6 months. In contrast, small neurons had immunoreactivity against cleaved caspase- 3 from age 3 months, suggesting that these neurons die by apoptotic activation.

In support of the protective role of NO, other work has shown that the inhibition of iNOS activity and iNOS knockout increases the brain inflammation and oligodendrocyte death in EAE [33]. In addition, the increased levels of iNOS in the cerebellum have been involved in the protection against oxidative stress caused by manganese intoxication [34]. Furthermore, the NO production mediated by nNOS together with BDNF has been associated with the maintenance of neurogenesis during the postnatal life [35].

It has been described that the NO-citrulline cycle participates in the differentiation of neural stem cells (NSC) into neurons, astrocytes, and oligodendrocytes, augmenting the $\beta$-3-tubillin and GFAP [35]. Based on this, we can suggest that NO participates in the astrogliosis manifested by an increased GFAP immunoreactivity [28]. On the contrary, other authors have documented the cytotoxic role of $\mathrm{NO}$ in the cerebellum. Accordingly, the nNOS and iNOS expressions were detected in the Purkinje layer after portacaval anastomosis or oxygen and glucose deprivation, thus suggesting that $\mathrm{NO}$ is involved in the pathology of these injury types [36].

Previous studies had shown that NO is able to produce lipoperoxidation [14] and mitochondrial injury in LPSstimulated oligodendrocytes [19,32]. In our work, we found enhanced NO production, iNOS and nNOS expression in oligodendrocytes, and lipoperoxidation in the cerebellum and brainstem of taiep rats. These results suggest that NO causing lipoperoxidation and mitochondrial injury might affect the myelin sheath, thus becoming one of the mechanisms responsible for the demyelination process of taiep animals. In support of this idea, the appearance of lipoperoxidation (at age 3 months) found in our work coincides with the appearance of demyelination in brain regions such as the spinal cord and diencephalon of the taiep rat [37].

In our work, we showed active caspase-3 IR in the cerebellum and brainstem of the SD rats and a higher IR intensity in those regions of the taiep rats at age 1 month. These results suggest that the caspase- 3 expression at younger ages is involved, at least in part, in the physiological process during the postnatal ontogeny of the brain. Accordingly, some reports have found that the content of caspase- 3 mRNA at postnatal day 40 in the cortex and lower levels in the brainstem did not directly correlate with variations in the number of brain cells [38]. In the taiep rat, the caspase- 3 immmunoreactivity was located in cells of the Purkinje layer, but not within the Purkinje cells. In this layer, the caspase-3 IR was located in small cells $(8-\mu \mathrm{m}$ diameter) neighboring to the Purkinje cells, suggesting that the Bergman cell bodies were inmunoreactive to cleaved caspase-3. It is possible that caspase-3 expressed in the Bergman cells might be involved in cell differentiation during the postnatal development of the cerebellum, as reported previously [31]. Some results provide evidence for a nontraditional role of caspases in cellular function that is independent of cell death and suggest that caspase activation is important for the astroglial cytoskeleton remodeling following cell injury [30]. Recently, a study in vitro has been reported that the presence of caspase- 3 in the neonatal rat astrocytes of two models of astrogliosis does not correlate with apoptosis [39]. Based on these reports, we can suggest that the presence of caspase- 3 in the cerebellum and brain stem of the taiep might be associated with astrogliosis, a key sign in taiep rats [28], rather than apoptosis.

In our work, the results with immunohistochemistry, Nissl counterstaining, and hematoxylin-eosin suggest that the Purkinje and magnocellular neurons in the old taiep rat died mainly by necrosis, which might be caused by lipoperoxidation. The increase in lipoperoxidation strongly suggests an increase in reactive oxygen species (ROS) and reactive nitrogen species (RNS), which might cause the rupture of the external mitochondrial membrane and consequently the activation of necrosis, as reported by other authors [40-42]. The progressive axonal degeneration in taiep rats, a chronic model with nonimmune-mediated demyelination [43], might account for the neuronal death in the cerebellum and brainstem of old taiep rats.

In summary, our results showed that the NO production that increases in an age-dependent manner in taiep rats might have a dual role; a protective role involved in the glial response to the hypomyelination-demyelination process, and a cytotoxic role mainly associated with demyelination (lipoperoxidation) and necrosis at least in the death of the Purkinje and magnocellular neurons. However, there are other factors that should be studied to gain insight into the taiep pathology, such as growth factor and cytokine activation that are important neurochemical responses in the myelin mutant taiep rats.

\section{ACKNOWLEDGEMENTS}

This work was supported by J-34143 (BAL-C) and 106694 (JRE) grants from CONACYT and VIEP-BUAP G/Nat/2012 (BAL-C) and G/SAL/2012 (JRE). Guadalupe Soto-Rodriguez and Rosa Arroyo was recipient of scholarships from CONACYT. We thank the Institute of 
Physiology of BUAP and CINVESTAV for the production and maintenance of both rats. Thanks to Dr. Ellis Glazier for the editing of the English-language text.

\section{REFERENCES}

[1] Holmgren, B., Urba-Holmgren, R., Riboni, L. and VegaSaenzdeMiera, E.C. (1989) Sprague dawley rat mutant with tremor, ataxia, tonic immobility episodes, epilepsy and paralysis. Laboratory Animal Science, 39, 226-228.

[2] Duncan, I.D., Lunn, K.F., Holmgren, B., Urba-Holmgren, R. and Brignolo-Holmes, L. (1992) The taiep rat: A myelin mutant with an associated oligodendrocyte microtubular defect. Journal of Neurocytology, 21, 870-884. doi:10.1007/BF01191684

[3] Lunn, K.F., Clayton, M.K. and Duncan, I.D. (1997) The temporal progression of the myelination defect in the taiep rat. Journal of Neurocytology, 26, 267-281. doi:10.1023/A:1018548400536

[4] Couve, E., Cabello, J.F., Krsulovic, J. and Roncagliolo, M. (1997) Binding of microtubules to transitional elements in oligodendrocytes of the myelin mutant taiep rat. Journal of Neuroscience Research, 47, 573-581. doi: $10.1002 /($ SICI) 1097-4547(19970315)47:6<573::AIDJNR2>3.0.CO;2-A

[5] Song, J., O’Connor, L.T., Yu, W., Baas, P.W. and Duncan, I.D. (1999) Microtubule alterations in cultured taiep rat oligodendrocytes lead to deficits in myelin membrane formation. Journal of Neurocytology, 28, 671-683. doi:10.1023/A:1007060832459

[6] Li, F.Y., Song, J. and Duncan, I.D. (2003) Mapping of taiep rat phenotype to rat Chromosome 9. Mammalian Genome, 14, 703-705. doi:10.1007/s00335-003-2302-z

[7] O'Connor, L.T., Goetz, B.D., Couve, E., Song, J. and Duncan, I.D. (2000) Intracellular distribution of myelin protein gene products is altered in oligodendrocytes of the taiep rat. Molecular and Cellular Neuroscience, 16, 396-407. doi:10.1006/mcne.2000.0889

[8] Leon-Chavez, B.A., Antonio Gonzalez-Barrios, J., Ugarte, A., Meraz, M.A. and Martinez-Fong, D. (2003) Evidence in vitro of glial cell priming in the taiep rat. Brain Research, 965, 274-278. doi:10.1016/S0006-8993(02)04143-4

[9] Leon-Chavez, B.A., Aguilar-Alonso, P., Gonzalez-Barrios, J.A., Eguibar, J.R., Ugarte, A., Brambila, E., Ruiz-Arguelles, A. and Martinez-Fong, D. (2006) Increased nitric oxide levels and nitric oxide synthase isoform expression in the cerebellum of the taiep rat during its severe demyelination stage. Brain Research, 1121, 221-230. doi:10.1016/j.brainres.2006.08.097

[10] Zhang, Y., Wang, H., Li, J., Jimenez, D.A., Levitan, E.S., Aizenman, E. and Rosenberg, P.A. (2004) Peroxynitrite-induced neuronal apoptosis is mediated by intracellular zinc release and 12-lipoxygenase activation. Journal of Neurocytology, 24, 10616-10627.

[11] Aguilar-Alonso, P., Martinez-Fong, D., Pazos-Salazar, N.G., Brambila, E., Gonzalez-Barrios, J.A., Mejorada, A., Flores, G., Millan-Perezpena, L., Rubio, H. and Leon-
Chavez, B.A. (2008) The increase in zinc levels and upregulation of zinc transporters are mediated by nitric oxide in the cerebral cortex after transient ischemia in the rat. Brain Research, 1200, 89-98. doi:10.1016/j.brainres.2007.11.077

[12] Zhou, J.F., Yan, X.F., Ruan, Z.R., Peng, F.Y., Cai, D., Yuan, H., Sun, L., Ding, D.Y. and Xu, S.S. (2000) Heroin abuse and nitric oxide, oxidation, peroxidation, lipoperoxidation. Biomedical and Environmental Sciences, 13, 131-139.

[13] Beckman, J.S., Beckman, T.W., Chen, J., Marshall, P.A. and Freeman, B.A. (1990) Apparent hydroxyl radical production by peroxynitrite: Implications for endothelial injury from nitric oxide and superoxide. Proceedings of the National Academy of Sciences of USA, 87, 1620-1624. doi:10.1073/pnas.87.4.1620

[14] Van der Veen, R.C. and Roberts, L.J. (1999) Contrasting roles for nitric oxide and peroxynitrite in the peroxidation of myelin lipids. Journal of Neurocytology, 95, 1-7.

[15] Ding-Zhou, L., Marchand-Verrecchia, C., Croci, N., Plotkine, M. and Margaill, I. (2002) L-NAME reduces infarction, neurological deficit and blood-brain barrier disrupttion following cerebral ischemia in mice. European Journal of Pharmacology, 457, 137-146. doi:10.1016/S0014-2999(02)02686-9

[16] Ortiz, G.G., Macias-Islas, M.A., Pacheco-Moises, F.P., Cruz-Ramos, J.A., Sustersik, S., Barba, E.A. and Aguayo, A. (2009) Oxidative stress is increased in serum from Mexican patients with relapsing-remitting multiple sclerosis. Disease Markers, 26, 35-39.

[17] Kwak, E.K., Kim, J.W., Kang, K.S., Lee, Y.H., Hua, Q.H., Park, T.I., Park, J.Y. and Sohn, Y.K. (2005) The role of inducible nitric oxide synthase following spinal cord injury in rat. Journal of Korean Medical Science, 20, 663669. doi:10.3346/jkms.2005.20.4.663

[18] Yao, S., Pandey, P., Ljunggren-Rose, A. and Sriram, S. (2010) LPS mediated injury to oligodendrocytes is mediated by the activation of nNOS: Relevance to human demyelinating disease. Nitric Oxide, 22, 197-204. doi:10.1016/j.niox.2009.12.001

[19] Yao, S.Y., Natarajan, C. and Sriram, S. (2012) nNOS mediated mitochondrial injury in LPS stimulated oligodendrocytes. Mitochondrion, 12, 336-344. doi:10.1016/j.mito.2012.01.002

[20] Hewett, S.J., Corbett, J.A., McDaniel, M.L. and Choi, D.W. (1993) Interferon-gamma and interleukin-1 beta induce nitric oxide formation from primary mouse astrocytes. Neuroscience Letters, 164, 229-232. doi:10.1016/0304-3940(93)90898-U

[21] Simmons, M.L. and Murphy, S. (1992) Induction of nitric oxide synthase in glial cells. Journal of Neurochemistry, 59, 897-905. doi:10.1111/j.1471-4159.1992.tb08328.x

[22] Merrill, J.E., Murphy, S.P., Mitrovic, B., MackenzieGraham, A., Dopp, J.C., Ding, M., Griscavage, J., Ignarro, L.J. and Lowenstein, C.J. (1997) Inducible nitric oxide synthase and nitric oxide production by oligodendrocytes. Journal of Neuroscience Research, 48, 372-384. doi:10.1002/(SICI)1097-4547(19970515)48:4<372::AIDJNR9>3.0.CO;2-8 
[23] Borgerding, R.A. and Murphy, S. (1995) Expression of inducible nitric oxide synthase in cerebral endothelial cells is regulated by cytokine-activated astrocytes. Journal of Neurochemistry, 65, 1342-1347. doi:10.1046/j.1471-4159.1995.65031342.x

[24] Soane, L., Rus, H., Niculescu, F. and Shin, M.L. (1999) Inhibition of oligodendrocyte apoptosis by sublytic C5b-9 is associated with enhanced synthesis of bcl-2 and mediated by inhibition of caspase-3 activation. Journal of Immunology, 163, 6132-6138.

[25] Hisahara, S., Okano, H. and Miura, M. (2003) Caspasemediated oligodendrocyte cell death in the pathogenesis of autoimmune demyelination. Neuroscience Research, 46, 387-397. doi:10.1016/S0168-0102(03)00127-5

[26] Ming, X., Li, W., Maeda, Y., Blumberg, B., Raval, S., Cook, S.D. and Dowling, P.C. (2002) Caspase-1 expression in multiple sclerosis plaques and cultured glial cells. Journal of the Neurological Sciences, 197, 9-18.

[27] Gonzalez-Barrios, J.A., Escalante, B., Valdes, J., LeonChavez, B.A. and Martinez-Fong, D. (2002) Nitric oxide and nitric oxide synthases in the fetal cerebral cortex of rats following transient uteroplacental ischemia. Brain Research, 945, 114-122. doi:10.1016/S0006-8993(02)02746-4

[28] Leon Chavez, B.A., Guevara, J., Galindo, S., Luna, J., Ugarte, A., Villegas, O., Mena, R., Eguibar, J.R. and Martinez-Fong, D. (2001) Regional and temporal progression of reactive astrocytosis in the brain of the myelin mutant taiep rat. Brain Research, 900, 152-155. doi:10.1016/S0006-8993(01)02284-3

[29] Sedmak, J.J. and Grossberg, S.E. (1977) A rapid, sensitive, and versatile assay for protein using Coomassie brilliant blue G250. Analytical Biochemistry, 79, 544-552. doi:10.1016/0003-2697(77)90428-6

[30] Acarin, L., Villapol, S., Faiz, M., Rohn, T.T., Castellano, B. and Gonzalez, B. (2007) Caspase-3 activation in astrocytes following postnatal excitotoxic damage correlates with cytoskeletal remodeling but not with cell death or proliferation. Glia, 55, 954-965. doi:10.1002/glia.20518

[31] Finckbone, V., Oomman, S.K., Strahlendorf, H.K. and Strahlendorf, J.C. (2009) Regional differences in the temporal expression of non-apoptotic caspase-3-positive bergmann glial cells in the developing rat cerebellum. Frontiers in Neuroanatomy, 3, 3.

[32] Arnett, H.A., Hellendall, R.P., Matsushima, G.K., Suzuki, K., Laubach, V.E., Sherman, P. and Ting, J.P. (2002) The protective role of nitric oxide in a neurotoxicant-induced demyelinating model. Journal of Immunology, 168, 427433.

[33] Dalton, D.K. and Wittmer, S. (2005) Nitric-oxide-dependent and independent mechanisms of protection from CNS inflammation during Th1-mediated autoimmunity: Evidence from EAE in iNOS KO mice. Journal of Neuroimmunology, 160, 110-121.

\section{doi:10.1016/j.jneuroim.2004.11.004}

[34] Chtourou, Y., Fetoui, H., Garoui, E.M., Boudawara, T. and Zeghal, N. (2012) Improvement of cerebellum redox states and cholinergic functions contribute to the beneficial effects of silymarin against manganese-induced neurotoxicity. Neurochemical Research, 37, 469-479. doi:10.1007/s11064-011-0632-x

[35] Lameu, C., Trujillo, C.A., Schwindt, T.T., Negraes, P.D., Pillat, M.M., Morais, K.L., Lebrun, I. and Ulrich, H. (2012) Interactions between the NO-citrulline cycle and BDNF in differentiation of neural stem cells. Journal of Biological Chemistry. doi:10.1074/jbc.M111.338095

[36] Suarez, I., Bodega, G., Rubio, M., Felipo, V. and Fernandez, B. (2005) Neuronal and inducible nitric oxide synthase expression in the rat cerebellum following portacaval anastomosis. Brain Research, 1047, 205-213. doi:10.1016/j.brainres.2005.04.035

[37] Moller, J.R., Durr, P.G., Quarles, R.H. and Duncan, I.D. (1997) Biochemical analysis of myelin proteins in a novel neurological mutant: The taiep rat. Journal of Neurochemistry, 69, 773-779. doi:10.1046/j.1471-4159.1997.69020773.x

[38] Kalinina, T.S., Bannova, A.V. and Dygalo, N.N. (2001) Content of apoptotic enzyme caspase-3 mRNA in brain stem and cortex in rats during postnatal ontogeny. Bulletin of Experimental Biology and Medicine, 132, 748-750. doi:10.1023/A:1013073710635

[39] Aras, R., Barron, A.M. and Pike, C.J. (2012) Caspase activation contributes to astrogliosis. Brain Research, 1450, 102-115. doi:10.1016/j.brainres.2012.02.056

[40] Baines, C.P., Kaiser, R.A., Purcell, N.H., Blair, N.S., Osinska, H., Hambleton, M.A., Brunskill, E.W., Sayen, M.R., Gottlieb, R.A., Dorn, G.W., Robbins, J. and Molkentin, J.D. (2005) Loss of cyclophilin D reveals a critical role for mitochondrial permeability transition in cell death. Nature, 434, 658-662. doi:10.1038/nature03434

[41] Nakagawa, T., Shimizu, S., Watanabe, T., Yamaguchi, O., Otsu, K., Yamagata, H., Inohara, H., Kubo, T. and Tsujimoto, Y. (2005) Cyclophilin D-dependent mitochondrial permeability transition regulates some necrotic but not apoptotic cell death. Nature, 434, 652-658. doi:10.1038/nature03317

[42] McConkey, D.J. and Orrenius, S. (1997) The role of calcium in the regulation of apoptosis. Biochemical and Biophysical Research Communications, 239, 357-366. doi:10.1006/bbrc.1997.7409

[43] Wilkins, A., Kondo, Y., Song, J., Liu, S., Compston, A., Black, J.A., Waxman, S.G. and Duncan, I.D. (2010) Slowly progressive axonal degeneration in a rat model of chronic, nonimmune-mediated demyelination. Journal of Neuropathology \& Experimental Neurology, 69, 12561269. doi:10.1097/NEN.0b013e3181ffc317 